\title{
The association between comorbidities and the quality of life among colorectal cancer survivors in the People's Republic of China
}

This article was published in the following Dove Press journal:

Patient Preference and Adherence

14 June 2016

Number of times this article has been viewed

\author{
Ji-Wei Wang ${ }^{1, *}$ \\ Li Sun ${ }^{1,2, *}$ \\ Ning Ding ${ }^{3}$ \\ Jiang $\mathrm{Li}^{4}$ \\ Xiao-Huan Gong' \\ Xue-Fen Chen' \\ Dong-Hui Yu ${ }^{5}$ \\ Zheng-Nian Luo ${ }^{6}$ \\ Zheng-Ping Yuan ${ }^{7}$ \\ Jin-Ming $\mathrm{Yu}^{\prime}$
}

'School of Public Health and Key Laboratory of Public Health Safety, Fudan University, Shanghai, ${ }^{2}$ Changzhou Center for Disease Control and Prevention, Changzhou, Jiangsu Province, People's Republic of China; ${ }^{3}$ Centre for Research and Action in Public Health, The University of Canberra, Canberra, ACT, Australia; ${ }^{4}$ Jonathan and Karin Fielding School of Public Health, University of California, Los Angeles, CA, USA; ${ }^{5}$ College of Clinical Medicine, Anhui Medical University, Hefei, Anhui Province, 'Shanghai Health Education Association,

${ }^{7}$ Shanghai Cancer Rehabilitation Club, Shanghai, People's Republic of China

*These authors contributed equally to this work
Correspondence: Jin-Ming Yu

School of Public Health, Key Laboratory of Public Health Safety, Fudan University, 130 Dong-An Road, Shanghai 200032,

People's Republic of China

Tel +862 I 54237868

Email jmy@fudan.edu.cn
Background: Cancer survivors with certain comorbidities had lower quality of life (QOL). This study was performed to investigate the prevalence of comorbidities and the association between comorbidities and the QOL among Chinese colorectal cancer survivors (CCS).

Methods: A cross-sectional study was conducted among 1,398 CCS between April and July 2013 in Shanghai, People's Republic of China. All the participants were asked to complete a simplified Chinese version of the European Organization for Research and Treatment quality of life version 3 questionnaire and questions on sociodemographic characteristics and comorbidities. In order to mitigate the bias caused by confounding factors, multiple linear regression models were employed to calculate the adjusted means of QOL scores.

Results: The proportion of participants without any comorbidity was only $20.2 \%$. The CCS with comorbidities except hypertension scored significantly lower on the European Organization for Research and Treatment quality of life version 3 questionnaire global health and functioning scales and Functional Assessment of Cancer Therapy-General scales but higher on the European Organization for Research and Treatment quality of life version 3 questionnaire symptom scores, indicating that they had poorer QOL, particularly for cardiovascular, respiratory, digestive, and musculoskeletal diseases.

Conclusion: There exists a significant association between comorbidities and QOL among Chinese CCS, and participants with comorbidities generally reported lower QOL scores. These findings suggested comprehensive care for CCS.

Keywords: colorectal cancer survivors, quality of life, comorbidities, cancer care

\section{Introduction}

There were 14.1 million new cancer cases, 8.2 million cancer deaths, and 32.6 million people living with cancer (within 5 years of diagnosis) around the world in 2012 . The number of new cancer cases is expected to increase to 24 million by 2035 . Colorectal cancer was the third most common cancer with nearly 1.4 million new cases in $2012 .{ }^{1}$ In many developing countries, such as the People's Republic of China, the prevalence of colorectal cancer has increased, while mortality rates for colorectal cancer have decreased in recent years. ${ }^{2-4}$ The quality of life (QOL) of colorectal cancer survivors (CCS) is undermined by the side effects of cancer and its treatments, and has become a fundamental task of oncological research. ${ }^{5-8}$ Assessment of QOL of long-term CCS to monitor and care for both medical and psychological consequences of their cancer treatment and rehabilitation is imperative.

Cancer survivors often have other medical conditions or illnesses, called comorbidities..$^{9,10}$ The burden of comorbidity is high, with up to $80 \%$ of CCS reporting 
at least one comorbidity. ${ }^{11,12}$ The most common comorbidities include cardiovascular diseases, musculoskeletal problems, lung or breathing problems, digestive diseases, diabetes mellitus, and depression. ${ }^{12-14}$

There are an increasing number of studies demonstrating that comorbidities have an additive impact on QOL among patients with several chronic diseases. For example, a higher number in comorbidities is associated with the deterioration of QOL, ${ }^{15-17}$ and different comorbidities have different impacts on QOL. ${ }^{18-20}$ And, several studies also found that comorbidities were associated with poorer QOL among cancer patients in countries other than the People's Republic of China. ${ }^{21,22}$ Despite the prevalence and burden of colorectal cancer in patients, to our knowledge there is little information regarding the associations between comorbidities and QOL among CCS. This is particularly true for CCS who have survived beyond the initial treatment period. QOL information on CCS is important if one wishes to evaluate the impact of the disease and its treatments beyond survival, and to understand how QOL is influenced by comorbidities. Understanding these factors can help the medical providers to better identify those who may be at greater risk of diminished functioning and improve targeted delivery of cancer management programs.

In this study, we investigated the prevalence of comorbidities in CCS and assessed whether comorbidities were negatively associated with QOL among CCS in the People's Republic of China. A better understanding of the association between comorbidities and QOL among CCS forms the basis for improving their cancer care and QOL.

\section{Methods}

\section{Recruitments}

Between April and July 2013, participants were recruited from 17 multicommunity cancer rehabilitation centers, all of which were affiliated to Shanghai Cancer Rehabilitation Club (SCRC), Shanghai, People's Republic of China, a nongovernment organization serving cancer survivors exclusively. This self-help support group aiming at improving the survivors' QOL regularly offers rehabilitation activities, including physical exercise, relaxation training, counseling, psychotherapy, and a variety of leisure activities. ${ }^{23}$ The criteria for study enrollment are: 1) being clinically diagnosed with primary colorectal cancer; 2) being able to read, write, and speak in Chinese; and 3) volunteering to participate in this study. A total of 2,102 CCS had registered in the SCRC by the end of 2012. All members of the SCRC who intended to continue to participate in the SCRC were required to register annually, including both former and new members. The survey invitations were sent through short text messages and/or phone calls to all the 2,102 CCS. Among all these CCS, 169 were unable to be reached because of death, migration, or refusing to respond; 554 failed to participate in the survey either because they had no time or because their health status or literacy ability was too poor. Finally, 1,398 participated in this survey and formed our final sample. Ethical approval to conduct this study was granted by the Medical Research Ethics Committee of the School of Public Health, Fudan University (protocol number RB \#2013-04-0450). A written informed consent was obtained from each participant.

\section{Instruments}

\section{Demographic characteristics}

The demographic characteristics included age, sex, body mass index, years since diagnosis, marital status, the highest educational level achieved, and monthly household income per capita.

\section{Comorbidities}

Participants were asked to indicate either "yes" or "no" on a list of comorbidities, including hypertension; diabetes mellitus; and cardiovascular, respiratory, digestive, and musculoskeletal diseases.

\section{QOL measurements}

The QOL was measured by the simplified Chinese version of the European Organization for Research and Treatment quality of life version 3 questionnaire (EORTC QLQ-C30). ${ }^{24}$ The EORTC QLQ-C30 core questionnaire contains five functional scales (physical, role, cognitive, emotional, and social), three symptom scales (fatigue, pain, and nausea and vomiting), a global health scale, a number of single items assessing additional symptoms commonly reported by cancer patients (dyspnea, appetite loss, insomnia, constipation, and diarrhea), and the financial impact of the disease. For most items, four response categories from 1 (not at all) to 4 (very much) are employed; two items (overall health and overall QOL) have response categories ranging from 1 to 7 . The scoring systems are organized such that a high scale score represents a higher response level. Thus, a high score for a functional scale, the global health status, or overall QOL represents a high or healthy status or a high QOL; however, a high score for the symptom scales represents a high level of symptomatology or problems. The scoring of the EORTC QLQ-C30 items was performed according to the EORTC 
scoring manual. In this study, the raw EORTC QLQ-C30 scores were transformed to scores ranging from 0 to $100 .{ }^{25}$

EORTC QLQ-C30 scales have shown good reliability in several different language versions as well as in simplified Chinese versions. ${ }^{26-29}$ In a study among Chinese cancer patients in which the reliabilities (as measured by Cronbach's alpha) of the scales were assessed on the first and second day of their being hospitalized, the results indicated that the alpha coefficients for all domains were $>0.7$, except for cognitive functioning (0.49). The test-retest reliability coefficients for most domains were $>0.80$, except for appetite loss $(0.77)$ and diarrhea $(0.75){ }^{29}$

\section{Statistical analysis}

Standard statistical methods were used to examine potential differences in covariates by one-way analysis of variance for continuous variables followed by Bonferroni post hoc tests. Comorbidity was defined in the statistical analyses as a dichotomous variable (yes/no). Multiple linear regression models were used to compute regression coefficients $(\beta)$ and associated $95 \%$ confidence intervals as estimates of the mean difference of QOL scores associated with the presence or absence of comorbidities, adjusting for potential confounding variables. The following potentially confounding variables were included in all regression models: age (continuous), body mass index (continuous), years since diagnosis (continuous), household income (continuous), education (less than junior high school, junior high school, or above junior high school), and current marital status (married/living with partner or divorced/widowed/separated/single). Tests for trend were performed by entering the categorical variables as continuous parameters in the model. Linear regression was applied to each of the variables measured in EORTC QLQ-C30. Comorbidity and other diseases (hypertension, diabetes mellitus, etc.) were not included in one model. Statistical tests were based on a two-tailed probability with a significance level of 0.05. Statistical analyses were performed using the Statistical Package for the Social Sciences for Windows (Version 21.0; IBM Corporation, Armonk, NY, USA).

\section{Results}

\section{The characteristics of the study sample}

The sociodemographic characteristics of the 1,398 participants (670 males and 728 females) and the prevalence of comorbidities in CCS are summarized in Table 1. The mean age was 64.5 years and average years since diagnosis were 8.6 years. Only one-fifth (20.2\%) of CCS reported no comorbidities. The most common comorbidity among CCS was
Table I Summary statistics of I,398 colorectal cancer survivors

\begin{tabular}{|c|c|}
\hline Variables & Number (\%) \\
\hline \multicolumn{2}{|l|}{ Age (years) } \\
\hline$<50$ & $45(3.2)$ \\
\hline $50-59$ & $343(24.5)$ \\
\hline $60-69$ & $649(46.4)$ \\
\hline$\geq 70$ & $361(25.8)$ \\
\hline \multicolumn{2}{|l|}{ Sex } \\
\hline Male & $670(47.9)$ \\
\hline Female & $728(52.1)$ \\
\hline \multicolumn{2}{|l|}{ Body mass index (BMI, $\mathrm{kg} / \mathrm{m}^{2}$ ) } \\
\hline$<18.5$ & $169(12.1)$ \\
\hline$\leq 18-<25$ & $735(52.6)$ \\
\hline$\leq 25-<30$ & $366(26.2)$ \\
\hline$\geq 30$ & $128(9.2)$ \\
\hline Years since diagnosis & $8.6 \pm 7.0$ \\
\hline \multicolumn{2}{|l|}{ Marital status } \\
\hline Divorced/widowed/separated/single & $158(11.3)$ \\
\hline Married/with partner & $\mathrm{I}, 240(88.7)$ \\
\hline \multicolumn{2}{|l|}{ Education } \\
\hline Primary school & $144(10.3)$ \\
\hline Middle school or vocational school & $1,005(71.9)$ \\
\hline Junior college or above & $249(17.8)$ \\
\hline \multicolumn{2}{|l|}{ Monthly household income per capita ( $¥)$} \\
\hline Under 2,000 & 348 (24.9) \\
\hline $2,000-4,000$ & $805(57.6)$ \\
\hline Over 4,000 & $245(17.5)$ \\
\hline \multicolumn{2}{|l|}{ Number of comorbidities } \\
\hline 0 & $283(20.2)$ \\
\hline 1 & $311(22.2)$ \\
\hline 2 & $277(19.8)$ \\
\hline$\geq 3$ & $527(37.7)$ \\
\hline \multicolumn{2}{|l|}{ Comorbidities } \\
\hline Hypertension & $538(38.5)$ \\
\hline Diabetes & $250(17.9)$ \\
\hline Cardiovascular & $360(25.8)$ \\
\hline Respiratory diseases & $169(12.1)$ \\
\hline Digestive diseases & $723(51.7)$ \\
\hline Musculoskeletal diseases & $409(29.3)$ \\
\hline
\end{tabular}

digestive diseases (51.7\%). Other comorbidities are listed according to their prevalence as follows: hypertension (38.5\%), diabetes mellitus (17.9\%), heart and cardiovascular diseases $(25.8 \%)$, respiratory diseases $(12.1 \%)$, and musculoskeletal diseases $(29.3 \%)$.

\section{The association between comorbidities and adjusted scores of QOL}

The association between comorbidities and adjusted scores of QOL is presented in Table 2. The results of multiple linear regression models showed that the participants with self-reported comorbidities generally reported lower scores for most EORTC QLQ-C30 scales when compared to the participants without these comorbidities. The influences of heart 


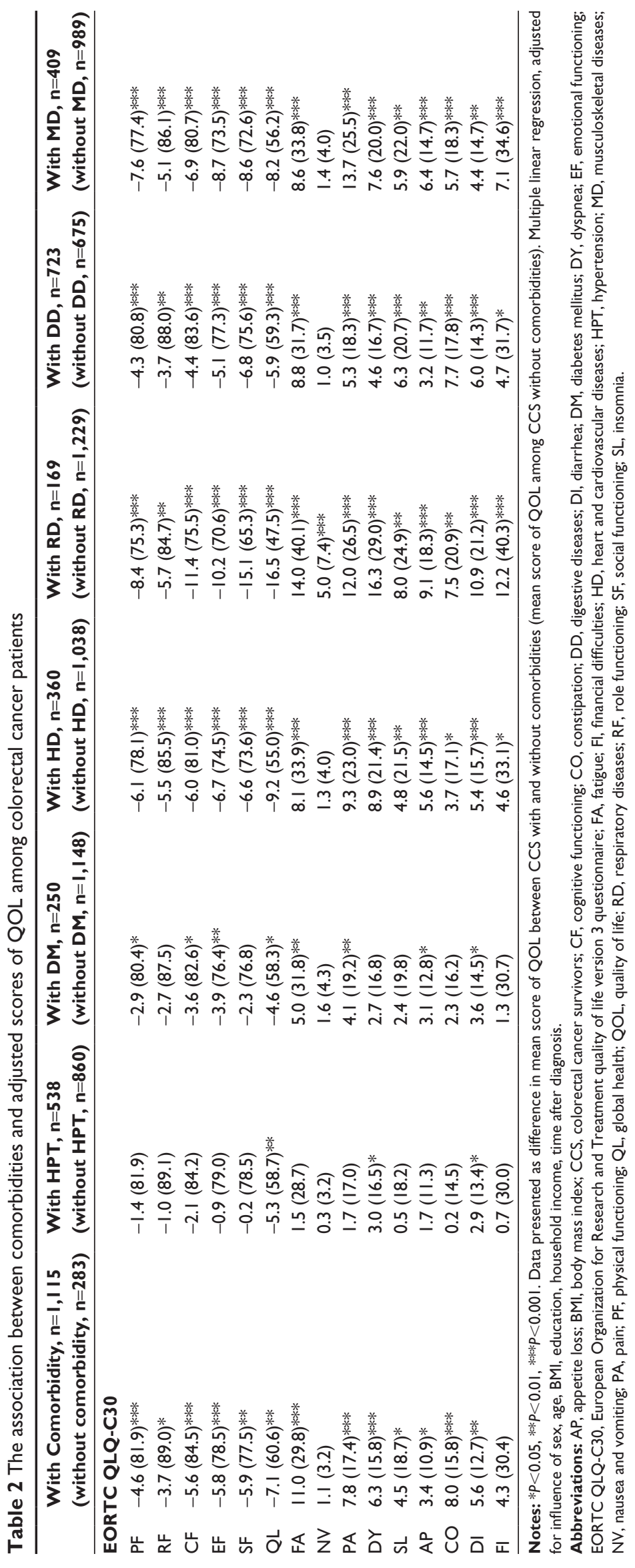


and cardiovascular diseases, respiratory diseases, digestive diseases, and musculoskeletal diseases on EORTC QLQ-C30 scores were of a similar magnitude and were larger than the influence of hypertension or diabetes mellitus.

\section{Discussion}

Based on our data, comorbidities, including hypertension, diabetes mellitus, heart and cardiovascular diseases, and respiratory, digestive, and musculoskeletal diseases, were quite common among CCS. This study showed that Chinese CCS with certain comorbidities such as cardiovascular, respiratory, digestive, and musculoskeletal diseases had lower QOL.

Previous studies suggested that age, income, educational level, marital status, and other variables had influence on QOL in cancer survivors. ${ }^{30,31}$ The main purpose of this study was to understand the impact of comorbidities on QOL; therefore, we used multiple linear regression models to control the effects of sociodemographic characteristics.

Cancer survivors with comorbidities have different clinical and health care needs than those without comorbidities. Moreover, cancer survivors tend to have more than one comorbidity. ${ }^{32,33}$ In our study, $\sim 80 \%$ of CCS had at least one comorbidity, and nearly $40 \%$ had more than three comorbidities. Noticeably, more than half of the CCS had digestive diseases, which was the most common comorbid condition in our sample. Therefore, in order to enhance QOL of CCS, more attention should be paid to their comorbidities, especially digestive disorders. It is crucial to implement a comprehensive management program for chronic diseases and address the overall issues rather than apply stopgap and piecemeal solutions.

Chronic diseases are the major causes of death and disability worldwide. Chronic diseases, such as cancer, heart disease, stroke, diabetes mellitus, and respiratory diseases, share major risk factors in the field of unhealthy lifestyles, including tobacco use, unhealthy diet, physical inactivity, and lack of access to preventive care that are beyond genetics and social inequalities. With rapid modernization in the People's Republic of China, changes in people's lifestyle have been blamed for the increase in the prevalence of colorectal cancer. ${ }^{34}$ High caloric intake and physical inactivity are suspected to be the associated factors of colorectal disease and known to often result in many other comorbidities, for example, diabetes mellitus, hypertension, dyslipidemia, coronary heart disease, gallbladder disease, arthritis, and constipation. ${ }^{35-38}$ Our previous studies have also clearly indicated that the comorbidities and unhealthy lifestyle have negative influence on QOL among cancer survivors. ${ }^{39,40}$ The CCS with or without comorbidities should build a healthier lifestyle.

The association between the comorbidities and colorectal cancer challenges the old oncology treatment paradigms that solely focus on the disease itself. Collaborative chronic disease management models emphasize the continuity of long-term care and a good relationship between the patient and the provider, in which the patient is empowered to take an active role in their ongoing care such as making lifestyle changes ${ }^{41,42}$ such models may be particularly appropriate to improve the QOL of cancer survivors.

Admittedly, there were some limitations in our study. First, all participants were recruited from one Cancer Rehabilitation Club, even though it has $\sim 13,500$ cancer survivor members from all 17 districts in Shanghai. We acknowledge that selection bias may affect the study results. Second, the information on cancer staging is not available, so we are unable to examine the association between cancer staging and QOL. Future study should collect information on cancer staging and conduct analysis to determine the relationship between cancer staging and QOL. Finally, the validity of self-reported comorbidities could be questionable. ${ }^{43}$ Participants were asked to answer the questions related to morbidities based on clinical diagnosis. These self-report comorbidity items could be a viable tool to collect comorbidity data. ${ }^{44}$ However, we still lack a mechanism to double check the correctness of answers. In addition, we did not report about those who had comorbidities, how many were treated, and how well the disease was controlled. Other issues were that cancer staging and the type of treatment received were not controlled in the model. The internal validity may be affected to a certain extent.

\section{Conclusion}

There exists an association between comorbidities and QOL among Chinese CCS, and participants with comorbidities generally reported lower QOL scores. These findings underscore the importance of collaborative chronic disease management for CCS.

\section{Acknowledgments}

We would like to thank the Shanghai Cancer Rehabilitation Club for supporting this study. We are very grateful to all CCS who willingly and generously gave their time. This study was supported by grants from the National Social Science Foundation of China (Award Number 13CSH076) and Shanghai Municipal Commission of Health and Family Planning (Award Number 20134068). 


\section{Disclosure}

The authors report no conflicts of interest in this work.

\section{References}

1. Ferlay J, Soerjomataram I, Dikshit R. Cancer incidence and mortality worldwide: sources, methods and major patterns in GLOBOCAN 2012. Int J Cancer. 2015;136(5):359-386.

2. Center MM, Jemal A, Ward E. International trends in colorectal cancer incidence rates. Cancer Epidemiol Biomarkers Prev. 2009;18(6): $1688-1694$.

3. Han X, Huang C, Zhao J, Ding Y, Zhang H, Cao G. Incidence and survival of colorectal carcinoma among permanent residents in Yangpu District of Shanghai, from 2002 to 2012. Zhonghua Liu Xing Bing Xue Za Zhi. 2014;35(3):289-294.

4. American Cancer Society. Cancer Facts and Figures, 2013. Atlanta, GA: American Cancer Society Inc; 2014.

5. Smith-Gagen J, Cress RD, Drake CM, Romano PS, Yost KJ, Ayanian JZ. Quality-of-life and surgical treatments for rectal cancer-a longitudinal analysis using the California cancer registry. Psychooncology. 2010; 19(8):870-878.

6. Wilson TR, Alexander DJ, Kind P. Measurement of health-related quality of life in the early follow-up of colon and rectal cancer. Dis Colon Rectum. 2006;49(11):1692-1702.

7. Ramsey SD, Berry K, Moinpour C, Giedzinska A, Andersen MR. Quality of life in long term survivors of colorectal cancer. Am J Gastroenterol. 2002;97(5):1228-1234.

8. Roila F, Cortesi E. Quality of life as a primary end point in oncology. Ann Oncol. 2001;12(Suppl 3):S3-S6.

9. Piccirillo JF. Importance of comorbidity in head and neck cancer. Laryngoscope. 2000;110(4):593-602.

10. Charlson ME, Pompei P, Ales KL, MacKenzie CR. A new method of classifying prognostic comorbidity in longitudinal studies: development and validation. J Chronic Dis. 1987;40(5):373-383.

11. Jansen L, Koch L, Brenner H, Ardnt V. Quality of life among longterm ( $\geq 5$ years) colorectal cancer survivors - systematic review. Eur J Cancer. 2010;46(16):2879-2888.

12. Pedrazzani C, Cerullo G, De Marco G, et al. Impact of age-related comorbidity on results of colorectal cancer surgery. World J Gastroenterol. 2009;15(45):5706-5711.

13. Yabroff KR, Lawrence WF, Clauser S, Davis WW, Brown ML. Burden of illness in cancer survivors: findings from a population-based national sample. J Natl Cancer Inst. 2004;96:1322-1330.

14. Trentham-Dietz A, Remington PL, Moinpour CM, Hampton JM, Sapp AL, Newcomb PA. Health-related quality of life in female longterm colorectal cancer survivors. Oncologist. 2003;8:342-349.

15. Gijsen R, Hoeymans N, Schellevis FG, Ruwaard D, Satariano WA, van den Bos GA. Causes and consequences of comorbidity: a review. J Clin Epidemiol. 2001;54(7):661-674.

16. Kriegsman DM, Deeg DJ, Stalman WA. Comorbidity of somatic chronic diseases and decline in physical functioning: The Longitudinal Aging Study Amsterdam. J Clin Epidemiol. 2004;57(1):55-65.

17. Fortin M, Lapointe L, Hudon C, Vanasse A, Ntetu AL, Maltais D. Multimorbidity and quality of life in primary care: a systematic review. Health Qual Life Outcomes. 2004;2:51.

18. Langelaan M, de Boer MR, van Nispen RM, Wouters B, Moll AC, van Rens GH. Impact of visual impairment on quality of life: a comparison with quality of life in the general population and with other chronic conditions. Ophthalmic Epidemiol. 2007;14(3):119-126.

19. Vissers PA, Thong MS, Pouwer F, Creemers GJ, Slooter GD, van de Poll-Franse LV. Prospectively measured lifestyle factors and BMI explain differences in health-related quality of life between colorectal cancer patients with and without comorbid diabetes. Support Care Cancer. 2016;24(6):2591-2601.

20. Vissers PA, Thong MS, Pouwer F, Zanders MM, Coebergh JW, van de Poll-Franse LV. The impact of comorbidity on health-related quality of life among cancer survivors: analyses of data from the PROFILES registry. J Cancer Surviv. 2013;7(4):602-613.
21. Schag CA, Ganz PA, Wing DS, Sim MS, Lee JJ. Quality of life in adult survivors of lung, colon and prostate cancer. Qual Life Res. 1994; $3(2): 127-141$.

22. Vacek PM, Winstead-Fry P, Secker-Walker RH, Hooper GJ, Plante DA. Factors influencing quality of life in breast cancer survivors. Qual Life Res. 2003;12(5):527-537.

23. Shanghai Cancer Rehabilitation Club. A brief introduction to the Shanghai Cancer Rehabilitation Cancer Club. Available from: http://www.shcrc. cn/webs/field_news.aspx?id=35. Accessed February 26, 2014.

24. Aaronson NK, Ahmedzai S, Bergman B, et al. The European Organization for Research and Treatment of Cancer QLQ-C30: a quality-of-life instrument for use in international clinical trials in oncology. $J$ Natl Cancer Inst. 1993;85(5):365-376.

25. Bottomley A. The EORTC Quality of Life Group. EORTC QLQ-C30 Scoring Manual. 3rd ed. Brussels: EORTC Publications; 2001.

26. Luo N, Fones CS, Lim SE, Xie F, Thumboo J, Li SC. The European Organization for Research and Treatment of Cancer Quality of Life Questionnaire (EORTC QLQ-c30): validation of English version in Singapore. Qual Life Res. 2005;14(4):1181-1186.

27. Arraras JI, Suárez J, Arias-de-la-Vega F, et al. The EORTC Quality of Life Questionnaire for patients with colorectal cancer: EORTC QLQCR29 Validation Study for Spanish patients. Clin Transl Oncol. 2011; 13:50-56.

28. Jocham HR, Dassen T, Widdershoven G, Halfens RJG. Reliability and validity of the EORTC QLQ-C30 in palliative care cancer patients. Cent Eur J Med. 2009;4(3):348-357.

29. Wan C, Meng Q, Yang Z, et al. Validation of the simplified Chinese version of EORTC QLQ-C30 from the measurements of five types of inpatients with cancer. Ann Oncol. 2008;19(12):2053-2060.

30. Montazeri A, Hole DJ, Milroy R, McEwen J, Gillis CR. Quality of life in lung cancer patients: does socioeconomic status matter? Health Qual Life Outcomes. 2003;1:19.

31. Jordhøy MS, Fayers P, Loge JH, Saltnes T, Ahlner-Elmqvist M, Kaasa S. Quality of life in advanced cancer patients: the impact of sociodemographic and medical characteristics. Br J Cancer. 2001;85: $1478-1485$.

32. Guralnik JM, LaCroix AZ, Everett DF, Kovar M. Aging in the Eighties: The Prevalence of Co-morbidity and Its Association With Disability. Hyattsville, Md: National Center for Health Statistics; Advance Data From Vital and Health Statistics, No. 170. Hyattsville, MD: National Center for Health Statistics; 1989.

33. Schellevis FG, van der Velden J, van de Lisdonk E, van Eijk JT, van Weel C. Comorbidity of chronic diseases in general practice. J Clin Epidemiol. 1993;46:469-473.

34. Goh KL. Changing trends in gastrointestinal disease in the Asia-Pacific region. J Dig Dis. 2007;8(4):179-185.

35. Whittemore AS, Wu-Williams AH, Lee M, et al. Diet, physical activity, and colorectal cancer among Chinese in North America and China. J Natl Cancer Inst. 1990;82(11):915-926.

36. Lyon JL, Mahoney AW, West DW, et al. Energy intake: its relationship to colon cancer risk. J Natl Cancer Inst. 1987;78(5):853-861.

37. Slattery ML, Abd-Elghany N, Kerber R, Schumacher MC. Physical activity and colon cancer: a comparison of various indicators of physical activity to evaluate the association. Epidemiology. 1990;1(6): 481-485.

38. Le Marchand L, Wilkens LR, Kolonel LN, Hankin JH, Lyu LC. Associations of sedentary lifestyle, obesity, smoking, alcohol use, and diabetes with the risk of colorectal cancer. Cancer Res. 1997;57(21): 4787-4794.

39. Wang JW, Gong XH, Ding N, et al. The influence of comorbid chronic diseases and physical activity on quality of life in lung cancer survivors. Support Care Cancer. 2015;23:1383-1389.

40. Wang JW, Sun L, Li J, et al. Comorbid chronic diseases and their associations with quality of life among gynecological cancer survivors. BMC Public Health. 2015;15:965.

41. FisherEB, BrownsonCA, O'Toole ML, Shetty G, Anwuri VV, Glasgow RE. Ecological approaches to self-management: the case of diabetes. $\mathrm{Am} \mathrm{J}$ Public Health. 2005;95:1523-1535. 
42. McCorkle R, Ercolano E, Lazenby M, et al. Self-management: Enabling and empowering patients living with cancer as a chronic illness. $C A$ Cancer J Clin. 2011;61:50-62.

43. Katz JN, Chang LC, Sangha O, Fossel AH, Bates DW. Can comorbidity be measured by questionnaire rather than medical record review? Med Care. 1996;34:73-84.
44. Sridharan S, Berdeprado J, Vilar E, Roberts J, Farrington K. A selfreport comorbidity questionnaire for haemodialysis patients. $B M C$ Nephrol. 2014;15:134

\section{Publish your work in this journal}

Patient Preference and Adherence is an international, peer-reviewed, open access journal that focuses on the growing importance of patient preference and adherence throughout the therapeutic continuum. Patient satisfaction, acceptability, quality of life, compliance, persistence and their role in developing new therapeutic modalities and compounds to optimize clinical outcomes for existing disease states are major areas of interest for the journal. This journal has been accepted for indexing on PubMed Central. The manuscript management system is completely online and includes a very quick and fair peer-review system, which is all easy to use. Visit http://www. dovepress.com/testimonials.php to read real quotes from published authors.

Submit your manuscript here: http://www.dovepress.com/patient-preference-and-adherence-journal 